\title{
Clinical Pathology Data from Cynomolgus Monkeys from China in which Diarrhea Was Observed during Quarantine
}

\author{
Yan-Wei LIU ${ }^{1,2)}$, Syusaku SUZUKI ${ }^{1)}$, Masatoshi KASHIMA²), Hiroshi TOKADO'), \\ Koichiro FUKUZAKI ${ }^{2}$, and Hiroaki MIYAJIMA ${ }^{2)}$
}

1)Department of Veterinary Medicine, Faculty of Agriculture, Kagoshima University, 1-21-24 Korimoto, Kagoshima 890-8580 and ${ }^{2}$ Shin Nippon Biomedical Laboratories Ltd., 2438 Miyanoura, Kagoshima 891-1394, Japan

\begin{abstract}
Shin Nippon Biomedical Laboratories, Ltd. (SNBL) imported and quarantined 3,148 cynomolgus monkeys (aged 2.5 to 6.5 years) from China in 2002. The hematology and blood biochemistry data obtained from these monkeys on Day 32 of quarantine were analyzed separately by sex [2,890 animals in which no abnormalities were observed during the 35-day quarantine period (normal group), and 258 animals which exhibited diarrhea 1 to 12 times (diarrhea group)]. The values obtained for all parameters were within the normal range (mean $\pm S D$ ), and no significant abnormalities were noted in either sex. The clinical pathology data from 11 animals (6 males and 5 females) exhibiting diarrhea repeatedly (10 to 12 times) were statistically analyzed, and significant differences were noted in PLT and ALP in both sexes. The PLT values of these animals were within the normal group mean \pm $2 S D$, and were considered within the normal range. A significant difference was noted in some individual ALP values (males: Nos. 2 and 3, females: Nos. 1, 3, and 4). The clinical pathology data obtained from the normal group in this study basically correspond to the widely reported results already obtained from healthy cynomolgus monkeys, from which it can be concluded that the cynomolgus monkeys from China were generally healthy and presenting no particular abnormality. The clinical pathology data from the normal group will serve as valuable baseline data for experimenters using cynomolgus monkeys.
\end{abstract}

Key words: clinical pathology, cynomolgus monkey, diarrhea

Shin Nippon Biomedical Laboratories, Ltd. (SNBL) imported 3,148 cynomolgus monkeys for experimental use from China in 2002, and these animals were quarantined for 35 days. In clinical examinations including body weight measurement, loss of appetite and/or diarrhea accompanying a decrease in body weight were observed once or from 2 to 12 times in 258 monkeys (8.2\%); however, no abnormal signs were observed in the remaining 2,890 animals. Marburg disease, Ebola hemorrhagic fever and herpes B virus were not clinically observed in any quarantined monkey, irrespective of whether or not diarrhea was observed, and culture examinations, tuberculin reaction tests, and serum antibody examinations were negative for salmonella, shigella, tuberculosis, simian type D retrovirus, and simian AIDS virus [2].

(Received 18 May 2007 / Accepted 25 October 2007)

Address corresponding: Y.W. Liu, Shin Nippon Biomedical Laboratories Ltd., 2438 Miyanoura, Kagoshima 891-1394, Japan 
Eighteen clinical hematology and blood biochemistry (hereafter clinical pathology) parameters were evaluated for each animal on Day 32 of quarantine. The primary purpose of these examinations was to ascertain whether changes in clinical values offered any indication which could predict or augment the results for clinical signs observations and culture and antibody examinations. The secondary purpose was to provide clinical pathology reference data from within a quarantine period, which is often required by cynomolgus monkey experimenters.

The examined animals were cynomolgus monkeys that SNBL imported from China in 2002 (1,564 males, $1,584$ females, total: 3,148$)$. Body weight at importation ranged from 2.0 to $7.5 \mathrm{~kg}$, and age ranged from 2.5 to 6.5 years. The animals were kept individually in stainless steel cages $[69$ (D) $\times 61(\mathrm{~W}) \times 75 \mathrm{~cm}(\mathrm{H})]$ in the quarantine facility, which is maintained at a temperature of $26 \pm 2{ }^{\circ} \mathrm{C}$, humidity of $50 \pm 10 \%$, and with a $12-\mathrm{h}$ dark-light cycle, and 15 air changes an hour. Solid food (Teklad Global, daily, 10:00 and 15:00,108 g per animal, about 450 cal.) and water were provided ad libitum. The progenitors of these cynomolgus monkeys originated from Vietnam; however, this line had been bred for more than two generations in an artificial environment in special facilities for experimental animals in China. Details are given below [2].

Blood (approximately $5 \mathrm{ml} /$ body) was drawn from the femoral vein with a syringe without anesthesia on Day 32 of quarantine (between 10:00 and 12:00, before feeding). A $1 \mathrm{ml}$ aliquot of blood was transferred to a Vacutainer tube, and treated with the anticoagulant EDTA-2K. Nine parameters, erythrocyte count (RBC), leukocyte count (WBC), hematocrit value (HCT), hemoglobin concentration (HGB), platelet count (PLT), mean corpuscular volume (MCV), mean corpuscular hemoglobin $(\mathrm{MCH})$, mean corpuscular hemoglobin concentration (MCHC), and mean platelet volume (MPV), were evaluated using a hematology system (ADVIA120: Bayer Diagnostic Manufacturing).

To prepare blood chemistry analysis samples, the 4 $\mathrm{ml}$ aliquots of the drawn blood were left at room temperature for 20 to $60 \mathrm{~min}$, then serum was obtained by centrifugation $(1,710 \times \mathrm{g}, 3,000 \mathrm{rpm}, 15 \mathrm{~min})$. The samples were analyzed for the following nine parameters using an automatic analyzer (JCA-BM8, JEOL Co., Ltd., Tokyo, Japan): aspartate aminotransferase (GOT), alanine aminotransferase (GPT), alkaline phosphatase (ALP), total bilirubin (TB), total protein (TP), total cholesterol (T-CHO), glucose (GLU), blood urea nitrogen (BUN), and creatinine $(\mathrm{Cr})$.

Most of the 258 animals exhibiting diarrhea (once to several times) during the 35-day quarantine period recovered in a few days, but thereafter diarrhea was observed repeatedly (10 to 12 times) in 11 animals (6 males and 5 females). These animals showed no serious symptoms, none died, and all recovered. Body weight was measured at the beginning and end of the quarantine period. At the end of the quarantine period, no abnormal changes were observed in the 258 animals exhibiting diarrhea during the period, and body weight in these animals increased on average by $0.04 \mathrm{~kg}$ in males and by $0.11 \mathrm{~kg}$ in females. A temporary loss of appetite was noted in less than $10 \%$ of animals in the first week from the initiation of quarantine; however, this change was not observed from the second week. This paper elucidates the clinical pathology data drawn from a total of 3,148 animals $[1,445$ males and 1,445 females that showed no abnormal clinical signs (normal group), and 123 males and 125 females that exhibited diarrhea at least once (diarrhea group) during the 35-day quarantine period]. The clinical pathology data from eleven animals (6 males and 5 females) repeatedly exhibiting diarrhea, although it was considered not to have resulted from an infectious disease, were analyzed by sex.

The clinical pathology data from the normal and diarrhea groups are expressed as mean $\pm \mathrm{SD}$ (standard deviation) by sex in Table 1 . No significant differences were noted in any parameters. Most values in the diarrhea group were within the normal group mean $\pm 1 \mathrm{SD}$, but a minority (platelet count, glucose, etc) fell within the normal group mean $\pm 2 \mathrm{SD}$. From these results, it was judged that the values obtained were within the normal range.

Table 1 (clinical values in the normal group) shows that no symptom of any of the eight target infectious diseases (Marburg disease, Ebola hemorrhagic fever, herpes B virus disease, salmonella, shigella, tuberculosis, simian D type retrovirus, and simian AIDS virus) was observed, nor was any other form of infection indicated. 
Table 1. Mean and standard deviation for clinical values from the normal and diarrhea groups

\begin{tabular}{|c|c|c|c|c|}
\hline & \multicolumn{2}{|c|}{ Males } & \multicolumn{2}{|c|}{ Females } \\
\hline & $\begin{array}{l}\text { Normal group } \\
(1,445 \text { heads })\end{array}$ & $\begin{array}{l}\text { Diarrhea group } \\
\quad(123 \text { heads })\end{array}$ & $\begin{array}{l}\text { Normal group } \\
(1,445 \text { heads) }\end{array}$ & $\begin{array}{l}\text { Diarrhea group } \\
\text { (135 heads) }\end{array}$ \\
\hline $\mathrm{RBC}\left(10^{6} / \mathrm{mm}^{3}\right)$ & $5.39 \pm 0.41^{1)}$ & $5.41 \pm 0.45$ & $5.17 \pm 0.41$ & $5.21 \pm 0.41$ \\
\hline WBC $\left(10^{3} / \mathrm{mm}^{3}\right)$ & $11.48 \pm 4.04$ & $13.33 \pm 5.75$ & $11.17 \pm 4.75$ & $9.92 \pm 3.05$ \\
\hline $\mathrm{HCT}(\%)$ & $40.19 \pm 3.02$ & $39.13 \pm 2.96$ & $39.13 \pm 3.02$ & $38.74 \pm 2.89$ \\
\hline $\mathrm{HGB}(\mathrm{g} / \mathrm{dL})$ & $12.62 \pm 0.88$ & $12.52 \pm 0.94$ & $12.27 \pm 0.93$ & $12.30 \pm 0.92$ \\
\hline $\operatorname{PLT}\left(10^{3} / \mathrm{mm}^{3}\right)$ & $359.65 \pm 92.36$ & $397.82 \pm 107.35$ & $361.52 \pm 90.43$ & $355.98 \pm 104.90$ \\
\hline MCV (fL) & $74.72 \pm 3.40$ & $72.46 \pm 2.80$ & $75.81 \pm 3.60$ & $74.38 \pm 3.17$ \\
\hline $\mathrm{MCH}(\mathrm{pg})$ & $23.48 \pm 1.26$ & $23.20 \pm 1.20$ & $23.78 \pm 1.26$ & $23.64 \pm 1.19$ \\
\hline $\mathrm{MCHC}(\mathrm{g} / \mathrm{dL})$ & $31.45 \pm 1.32$ & $32.02 \pm 1.15$ & $31.38 \pm 1.09$ & $31.80 \pm 0.95$ \\
\hline MPV (fL) & $8.88 \pm 1.52$ & $8.34 \pm 1.43$ & $8.95 \pm 1.41$ & $8.80 \pm 1.35$ \\
\hline GOT (IU/L) & $33.80 \pm 12.86$ & $36.10 \pm 23.72$ & $34.35 \pm 14.25$ & $33.61 \pm 9.90$ \\
\hline GPT (IU/L) & $37.52 \pm 24.90$ & $43.66 \pm 32.14$ & $48.68 \pm 47.36$ & $39.85 \pm 29.25$ \\
\hline ALP (IU/L) & $988.80 \pm 389.13$ & $876.90 \pm 311.64$ & $657.671 \pm 332.73$ & $648.29 \pm 319.16$ \\
\hline $\mathrm{TB}(\mathrm{mg} / \mathrm{dL})$ & $0.14 \pm 0.04$ & $0.12 \pm 0.02$ & $0.16 \pm 0.06$ & $0.14 \pm 0.04$ \\
\hline $\mathrm{TP}(\mathrm{g} / \mathrm{dL})$ & $7.13 \pm 0.47$ & $7.07 \pm 0.58$ & $7.14 \pm 0.50$ & $6.88 \pm 0.51$ \\
\hline T_CHO (mg/dL) & $114.38 \pm 23.75$ & $114.56 \pm 27.76$ & $122.71 \pm 25.06$ & $117.16 \pm 21.48$ \\
\hline GLU (mg/dL) & $70.21 \pm 14.39$ & $65.13 \pm 10.62$ & $65.11 \pm 14.08$ & $59.95 \pm 10.25$ \\
\hline BUN (mg/dL) & $24.56 \pm 4.97$ & $25.23 \pm 5.57$ & $22.61 \pm 5.71$ & $19.51 \pm 5.08$ \\
\hline Cr. $(\mathrm{mg} / \mathrm{dL})$ & $0.55 \pm 0.15$ & $0.50 \pm 0.11$ & $0.47 \pm 0.09$ & $0.46 \pm 0.11$ \\
\hline
\end{tabular}

The test item abbreviation: JSCC. ${ }^{1)}$ : mean \pm SD.

During the 1980s, Sugimoto et al., [3], Yoshida [5] and Yoshida et al., [6-9] of the Tsukuba Primate Center undertook detailed investigations of clinical pathology data from cynomolgus monkeys varying factors such as age, sex, and rearing environment. Schuurman and Smith [4], and Koga et al., [1] have also published reports recently. The clinical pathology data obtained from the normal group in this study basically corresponds to those obtained from the healthy monkeys reported in these extensive references, from which it can be concluded that these cynomolgus monkeys from China were generally healthy and were presenting no particular abnormality.

The clinical pathology data from the animals (6 males and 5 females) exhibiting diarrhea repeatedly (10 to 12 times) during the 35-day quarantine period were statistically analyzed against mean values in the normal group by radar chart, and significant differences were noted in platelet count (PLT) and alkaline phosphatase (ALP) in both sexes (refer to Fig.1). PLT values in these animals were within the normal group mean $\pm 2 \mathrm{SD}$ (males: $359.65 \pm 184.72$; females: $361.52 \pm 180.86$ ), and were considered to be within the normal range. A significant difference was noted in some individual ALP values (males: Nos. 2 and 3; females: Nos. 1, 3, and 4).

It is assumed that frequent diarrhea accompanied by loss of appetite led to deterioration in nutritional state although this cannot be substantiated beyond doubt because the causes of diarrhea were varied. It is highly probable that the diarrhea was caused by stress and physiological changes resulting from fatigue from being transported to the quarantine facility in Kagoshima from the breeding facility in southwest China, or the changed rearing conditions. Accordingly, because this diarrhea was a transient change occurring at an early stage of the quarantine period, it was not considered to have resulted from any infectious disease. Differences in the clinical pathology data were considered related to the diarrhea, but their explanation remains uncertain. These changes were considered incidental because the number of cases was small but further investigation is required for clarification.

If clinical pathological examination had been repeatedly performed at regular intervals during the quarantine period, it could have been ascertained whether some kind of abnormality was being indicated, or cynomolgus monkeys quarantined were initially healthy. In either case, more data would have been required because the number 

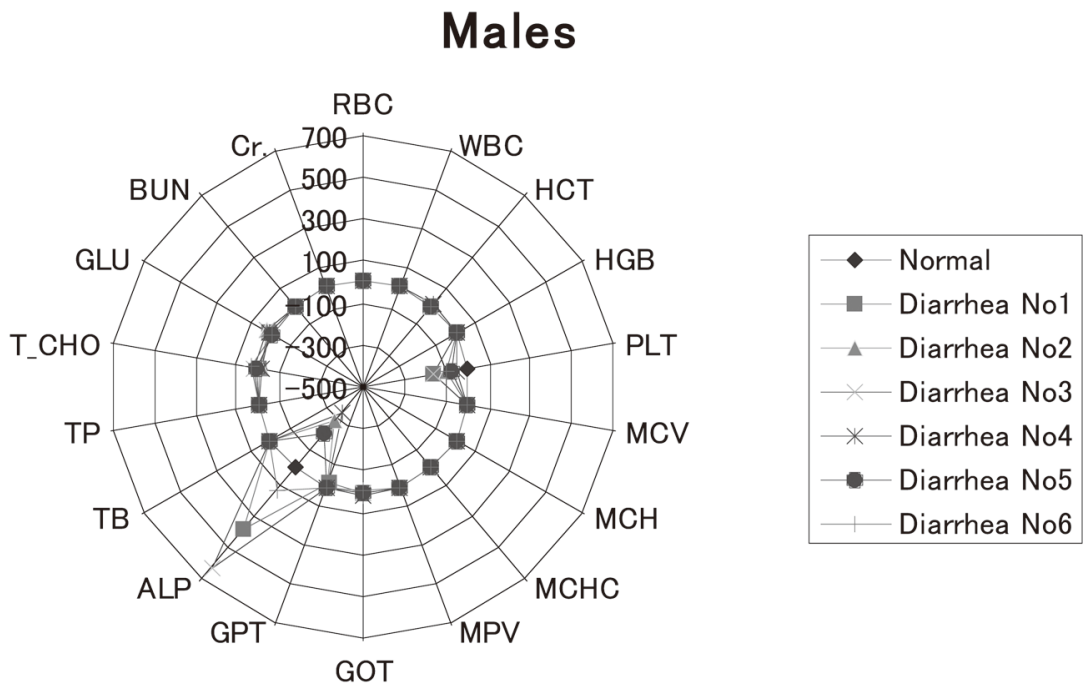

\section{Females}
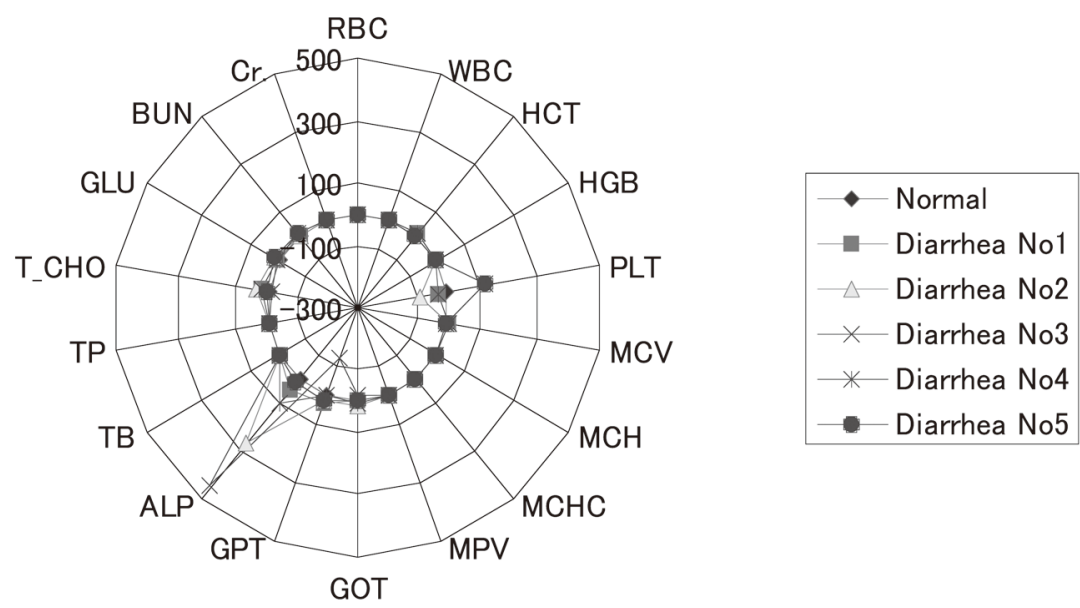

Fig. 1. The clinical pathology data from the animals (6 males and 5 females) exhibiting diarrhea repeatedly (10 to 12 times) during the 35-day quarantine period were statistically analyzed against mean values in the normal group by radar chart.

of animals in the diarrhea group was small compared with that in the normal group. Over 3,000 imported monkeys are quarantined every year at SNBL, and accumulation and analysis of all data obtained will continue. This issue will be reported further.

Yoshida [5] has stated that the physiology of cynomolgus monkeys stabilizes three months after importation. This information is important to importers of monkeys for experimentation, and it is intended that examinations continue beyond the quarantine period.
However, the cynomolgus monkeys imported by SNBL have been bred in the same environment in the Chinese facilities for over two generations, and it is concluded that there are no problems with the regular medical examinations performed there. After import into Japan, the animals are isolated in a strictly controlled environment, and it is considered that clinical examination values are already stabilized by Day 32 of quarantine, after one month of being reared in this environment.

The number of animals subject to clinical pathology 
examination, 1,445 males and 1,445 females in the normal group, was large. Therefore, it is strongly believed that the results obtained in this study are of value to experimenters using cynomolgus monkeys.

\section{Acknowledgment(s)}

We wish to thank professor Yasuhiro Yoshikawa of the Department of Biomedical Science, Graduate School of Agricultural and Life Science, the University of Tokyo, for his guidance on this paper.

\section{References}

1. Koga, T., Kanefuji, K., and Nakama, K. 2005. Int. J. Toxicol. 24: 377-385.

2. Liu, Y.W., Sakamoto, H., Tokado, H., Fukuzaki, K., and Miyajima, H. 2007. J. Jpn. Vet. Med. Assoc. 60: 809-812.

3. Sugimoto,Y., Hanaru, K,. Narita, H., and Honjo, S. 1986. Exp. Anim. 35: 443-447.

4. Schuurman, H.-J. and Smith,H.T. 2005. Xenotransplantation 12: 72-75.

5. Yoshida, T. 1981. Jpn. J. Med. Sci. Biol. 43: 239-242.

6. Yoshida, T., Suzaki, K., Cho, F., and Honjo, S. 1986. Exp. Anim. 35: 329-338.

7. Yoshida, T., Ohkubo, F., Ohtoh, H., and Honjo, S. 1986. Exp. Anim. 35: 449-454.

8. Yoshida, T., Ohtoh, K., Cho, F., and Goto, N. 1988. Exp. Anim. 37: 39-44.

9. Yoshida, T., Ohtoh, K., Cho, F., and Goto, N. 1990. Exp. Anim. 39: 21-26. 